\title{
A Dialogue on Dynamical Pre-Main Sequence Tracks
}

\author{
Günther Wuchterl \\ Max-Planck-Institute für extraterrestrische Physik, Postfach 1312, \\ 85741 Garching, Germany, wuchterl@mpe.mpg.de
}

\begin{abstract}
Based on the theory of stellar structure and evolution combined with the theory of stellar atmospheres theoretical properties of young stars can be calculated. These calculations of pre-main sequence evolution have been refined over the last decades and do now provide theoretical spectra and colours even for very cool objects like young stars brown dwarfs and planets. Two of their key assumptions must become invalid towards the formation phases: (1) the hydrostatic equilibrium of pressure forces and gravity that assumes stellar matter to be at rest and (2) the non-dependence on the initial thermal structure. The former (1) is violated by accretion- and collapse flows, the latter (2) because a new born young star is observed with the specific thermal structure produced by the cloud collapse. I discuss changes in the theoretical properties of young stars that follow from calculating the pre-main sequence evolution as the consequence of the collapse of Bonnor-Ebert spheres.
\end{abstract}

\section{A Fictional but Scientific Debate}

Dynamico: We have now calculated the properties of young stars that are produced by the protostellar collapse of Bonnor-Ebert spheres, (Wuchterl and Tscharnuter 2000). There are a few surprises even in the case of one solar mass: the young stars are not fully convective when they become visible for the first time and almost all the deuterium has been burnt during the main accretion phase. Look at the resulting Hertzsprung-Russel-diagram (Fig. 1): it shows the properties of the star during all the phases until mass accretion fades and the final stellar photosphere becomes visible.

Statica: This is interesting for the star formation process, if we put aside for the moment that you use spherical symmetry and hence you do not include disk accretion and that you ignore magnetic fields and hence you have no outflows. But as you know Hayashi (1961) and others have considered whether the star formation process will have any effect on the initial conditions for stellar evolution calculations and the properties of young stars in their first few million years. The result was that stars should become fully convective once they are hydrostatic. It does not matter how much entropy or, if you prefer, energy you put into your initial model, the details will be quickly forgotten.

Dynamico: I think this result, i.e. that the memory of the intial state is quickly lost, is due to the fact that a fully convective structure is assumed from the beginning. A fully convective structure requires the luminosity to be larger 


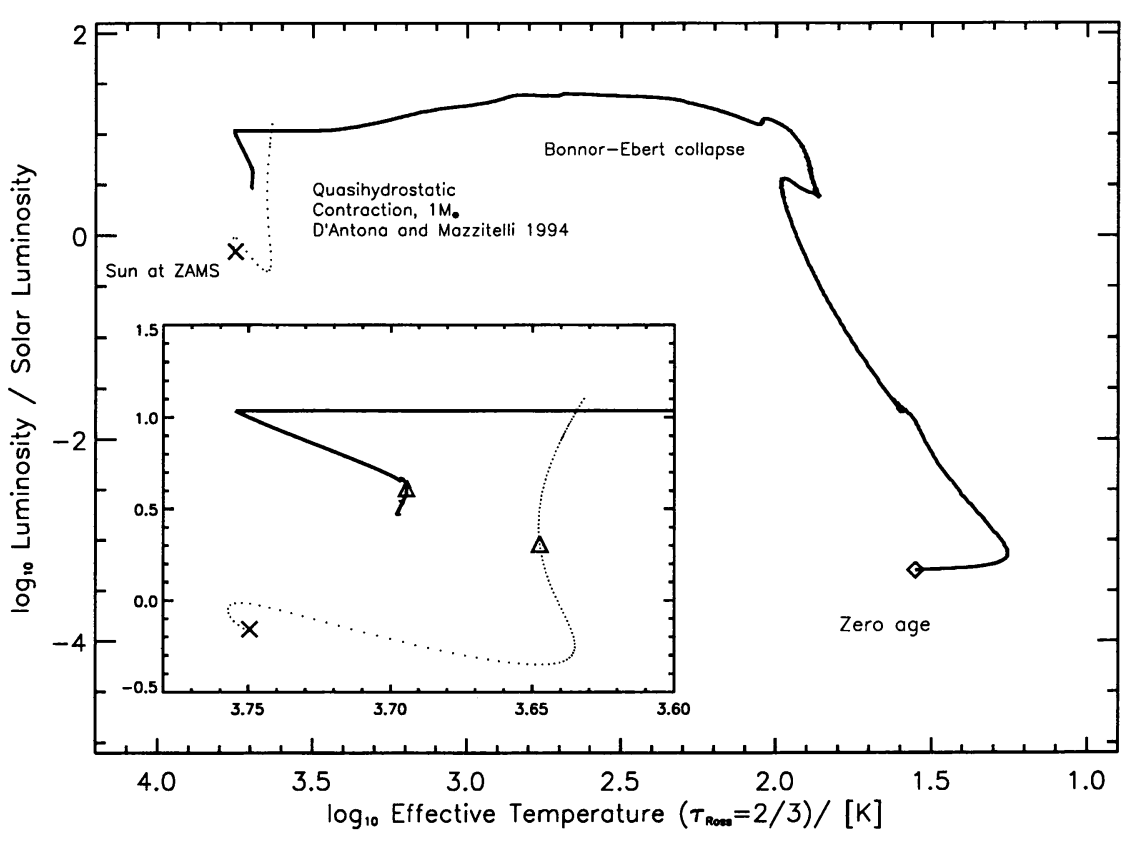

Figure 1. Hertzsprung-Russell diagram for the collapse and early evolution of solar mass stars. Comparison of a radiationhydrodynamical calculation of the collapse of a solar mass BonnorEbert sphere (Wuchterl and Tscharnuter 2000) and a classical stellar evolution calculation (D'Antona and Mazzitelli 1994). The equations of the latter are a hydrostatic limiting case of the former, including the convection prescription. Triangles mark ages of $1 \mathrm{Myr}$. See Wuchterl and Tscharnuter 2000, for details.

than the luminosity that can be transferred by radiation alone, everywhere in the star. Hence the luminosities tend to be large, and large luminosities quickly change the thermal structure. Energy transfer is very efficient once convection has started. In my results the stars have relatively cold centers and the temperature peaks off-center. That is why deuterium burns in a shell and convection is typically only present in the outer third of the radius, which corresponds to about half of the mass. Therefore luminosities are small, even negative in some parts of the young star. In such a situation with small and reversed temperature gradients convection cannot play its role to erase the thermal memory of the protostar so efficiently.

Statica: But look at the values that you predict for the case of a solar mass. They are hundreds of Kelvins hotter than everybody else found. To my eye they look like the result of neglecting the important effects of non-grey radiative transfer. And for cool objects these effects certainly have to be included.

Dynamico: You are certainly right that photospheric radiative transfer is a key to accurately calculate the stellar properties once the stars have settled into 
hydrostatic equilibrium and all sorts of accretion, circumstellar and other young star activity has stopped, say beyond $0.1 \mathrm{Gyr}$ or so. But as we go to progressively younger ages additional effects complicate the description of the photospheres. There are spots, i.e. a nonhomogeneous surface temperature distribution, there is veiling, i.e. additional contributions to the spectrum, and some luminosity comes from non-static parts of the photosphere, the change of mass with time because of accretion, and the 'residual' circumstellar matter that causes effects like internal reddening. Many of those effects are by nature only included in the dynamical calculations, admittedly in a simple way. But together they produce a photosphere that is not (yet) like a classical one. It is extended, i.e. non-plane parallel, gas is moving at low optical depth, i.e. the photosphere is dynamical, there is residual accretion on top of it, and matter flowing through it, and convection extending into it. To give an idea about the importance of these effects let me just mention that for $1 \mathrm{M}_{\odot}$ at $1 \mathrm{Myr}$ my effective temperature is about 500 Kelvin higher than the one you obtained. But my 'photospheric' temperature, i.e. the matter temperature at the radius where photons typically are radiated away is comparable to the 'classical' $T_{\text {eff }}$ values. This illustrates the fact that in dynamical convective photospheres, it is not necessarily true that $T_{\text {eff }}=T\left(\tau_{\text {Ross }}=2 / 3\right)$, even in the grey Eddington approximation.

I agree with you that grey radiative transfer can only be approximative but my point is that the remaining effects due to the collapse are dominating in the first million years. That is why I used a grey study (D'Antona and Mazzitelli 1994) as my reference. Everything that goes into the D'Antona and Mazzitelli track, including the value of the mixing length and the opacities corresponds to a hydrostatic limit of my equations. Yet the star I obtain following the collapse has different properties for as long as I calculate. Non-grey effects or an improved convection theory may change that result. But those corrections will only add to my present result.

Statica: You are talking about your time-dependent convection theory that you need in the dynamical regime of the protostellar collapse and accretion. We have also looked at the convection issue in some detail recently (and our point is that there should not be a shut-off of convective energy transfer at a certain optical depth in the atmosphere but that convective and radiative flux should be budgeted in the energy equation throughout the atmosphere). We think that the mixing length values that lead to the tracks that are now favoured by observers are the ones that we expect to be the physically correct ones. In any case we know what we are doing when we use mixing length theory. It is not a fancy theory but its behavior is well studied in stellar structure and atmospheric models. Your convection model on the other hand goes far into a region where it has never been tested observationally. Actually there is a whole brand of different convection theories that share a similar formalism and reasoning as yours. And there are indications that this type of convection theories has trouble in explaining the Cepheid pulsations.

Dynamico: You are right, the time dependent convection theory (Wuchterl and Feuchtinger 1999) required a substantial step beyond conventional mixing length theory. But to make sure we know how it is related to the rest of astrophysics it was constructed in a way that it approximates mixing length theory very closely in the hydrostatic limit. That has been checked by comparing the 
resulting convection zone for the Sun to a standard solar model that has been tested by helioseismology. The Sun is also used to fix the mixing length parameter. After that all our equations are completely determined. The so obtained equations have been succesfully tested by calculating RR Lyrae stars. What remains to be specified for the star formation problem are the conditions of the cloud at the onset of collapse. We have chosen a marginally stable Bonnor-Ebert sphere. After that there is no way to tune the results. Hence there is also no freedom to shift the PMS-tracks in temperature by changing the mixing length parameter. Therefore I think the temperatures for every mass and age can be tested by observation. Unlike the usual PMS-tracks that are published for various values of the MLT-paramter. If for a given observation different tracks for different MLT-parameters are considered I do not consider that an observational test because not all the freedom in the theory has been fixed before. It is more like an observational determination of a special mixing length parameter for young stars.

Empirico: This is philosophy, not science. If it fits it fits and the underlying model is OK, period! GG Tau fits the dynamical mass constraint on A+B and all four components are on an isochrone for Statica's tracks and hence they successfully pass the observational test.

Dynamico: This conclusion is true for a theory that is overconstrained in the sense that observational facts determine more properties than can be adjusted by using uncertainties in the theory. We still have to fix the parameters of the convection prescription before a stellar structure can be calculated. If that is not fixed by requiring the Sun to come out correctly, as is the case for GG Tau's favorite tracks, its not a test. The Sun would then be the test.

I am not saying my tracks must be correct in the sense that they are the most realistic ones, I just want to caution that before we draw the conclusions about the importance of birth-effects we should make sure that we have calibrated uncertain physical ingredients such as the convection prescriptions in the 'easy' mature stars and that we can distinguish between calibration of an observational

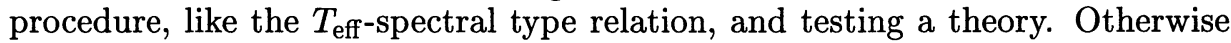
we might be calibrating the spectral type conversion instead of testing the tracks.

And one thing is certain: the protostellar collapse is not a hydrostatic process. And if that process is calculated dynamically in spherical symmetry with equations that contain a correct model of the Sun as a limiting case, the results are the ones that I presented im my Fig. 1.

\section{References}

Wuchterl, G., \& Tscharnuter, W. M. 2000, A\&A, submitted

Hayashi, C. 1961, PASJ, 13, 450

D'Antona, F., \& Mazzitelli, I. 1994, ApJS, 90, 467

Wuchterl, G., \& Feuchtinger M. U. 1998, A\&A, 340, 419 